Assurances et gestion des risques

Insurance and Risk Management

\title{
Comment construire un générateur de scénarios économiques risque neutre destiné à l'évaluation économique des contrats d'épargne ?
}

\author{
Kamal Armel et Frédéric Planchet
}

Volume 85, numéro 1-2, juin 2018

URI : https://id.erudit.org/iderudit/1051315ar

DOI : https://doi.org/10.7202/1051315ar

Aller au sommaire du numéro

Éditeur(s)

Faculté des sciences de l'administration, Université Laval

ISSN

1705-7299 (imprimé)

2371-4913 (numérique)

Découvrir la revue

Citer ce document

Armel, K. \& Planchet, F. (2018). Comment construire un générateur de scénarios économiques risque neutre destiné à l'évaluation économique des contrats d'épargne ? Assurances et gestion des risques / Insurance and Risk Management, 85(1-2), 27-55. https://doi.org/10.7202/1051315ar

Tous droits réservés (C) Faculté des sciences de l'administration, Université Laval, 2018
Ce document est protégé par la loi sur le droit d'auteur. L’utilisation des services d'Érudit (y compris la reproduction) est assujettie à sa politique d'utilisation que vous pouvez consulter en ligne.

https://apropos.erudit.org/fr/usagers/politique-dutilisation/ 


\section{COMMENT CONSTRUIRE UN GÉNÉRATEUR DE SCÉNARIOS ÉCONOMIQUES RISQUE NEUTRE DESTINÉ À L'ÉVALUATION ÉCONOMIQUE DES CONTRATS D'ÉPARGNE?}

\section{Kamal ARMEL ${ }^{\text {, }}$ Frédéric PLANCHET²}

Ce papier présente une démarche de construction d'un générateur de scénarios économiques risque neutre, destinés à l'évaluation du best-estimate des contrats d'épargne, dans le cadre d'un environnement économique caractérisé par des taux négatifs.

\section{TABLE DES MATIÈRES}

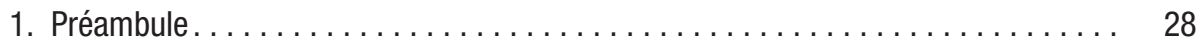

2. Générateur de scénarios économiques pour calculer le best-estimate ......... 29

2.1 Un cadre de valorisation Mark to Model . . . . . . . . . . . . . . . . . . . . . 29

2.2 La convention de calibrage des modèles de taux sans risque........... 30

2.3 Quelques limites de la convention de calibrage des modèles de taux sans risque pour l'évaluation du best-estimate. ................. 32

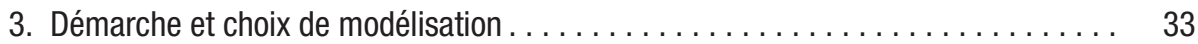

4. Présentation des données des modèles de taux................. 35

4.1 Analyse des taux sans risque du marché et de l'EIOPA . ........... 35

4.2 Volatilités implicites des caps et des swaptions ................. 36

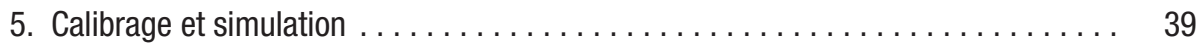

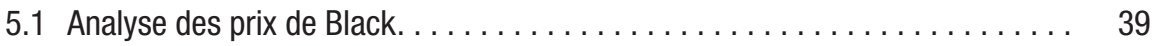

5.2 Modèle Hull \& White . . . . . . . . . . . . . . . . . . . . . . 40

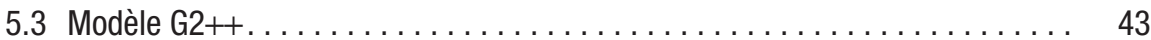

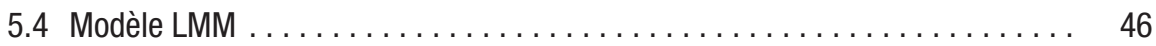

5.4.1 Étude du modèle LMM dans un cadre market-consistent......... 46

5.4.2 Etude du modèle LMM dans un cadre non market-consistent. . . . . . 48

5.5 Structure de dépendance et modèles actions et immobilier . . . . . . . . . . . 50

6. Conclusions ....................................... 52

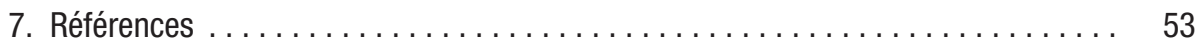




\section{Préambule}

Comme précisé dans l'annexe technique de ce papier, le processus de génération de scénarios économiques peut être synthétisé en trois étapes (Laurent et al. [2016], Planchet et al. [2009], Baldvinsdóttir et Palmborg [2011], Ballotta [2004], Armel et al. [2011]...):

1. L'environnement de modélisation: il s'agit de choisir les variables économiques à modéliser. Classiquement, la mesure retenue est une probabilité risque neutre.

2. Les modèles: il s'agit de construire les modèles mathématiques des variables d'intérêt. Cela consiste à choisir les modèles qui vont représenter la dynamique individuelle de ces variables et le choix du modèle qui représente le co-mouvement.

3. Les paramètres et le calibrage: il s'agit de choisir les produits financiers dérivés pour les calibrages, les données, les méthodes d'estimation statistique des paramètres des modèles et des méthodes de validation.

Ces étapes peuvent se schématiser comme présenté dans la Figure 1.

FIGURE 1 Illustration schématique des choix de modélisation et de calibrage d'un GSE
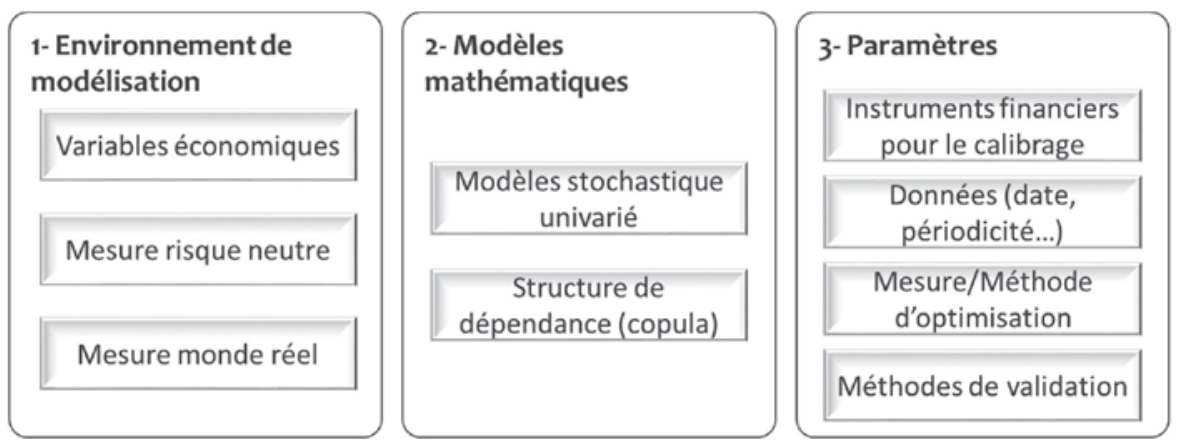

Ce papier présente une démarche de construction d'un GSE destiné à l'évaluation de la valeur économique (best-estimate) des contrats d'épargne, notamment les contrats en $€$. Les modèles mathématiques et les caractéristiques techniques du GSE utilisé dans la suite sont documentés dans l'annexe technique qui suit cet article. 
Les variables d'intérêt étudiées dans la suite sont les taux d'intérêt sans risque, les actions et les investissements en immobilier. Le risque de crédit n'est pas abordé ici.

Les prix des actions et des investissements en immobilier sont supposés suivre un mouvement brownien de type Black et Scholes et trois modèles de taux sont étudiés:

- Un modèle mono-factoriel: Hull \& White calibré sur des caps et sur des swaptions;

- Un modèle à deux facteurs: modèle gaussien G2++ calibré sur des caps et sur des swaptions;

- Un modèle de marché: Libor Market Model (LMM) calibré sur des swaptions.

La structure de dépendance retenue est la corrélation.

\section{GÉNÉRATEUR DE SCÉNARIOS ÉCONOMIQUES POUR CALCULER LE BEST-ESTIMATE}

\subsection{Un cadre de valorisation Mark to Mode/ ${ }^{3}$}

Les scénarios économiques utilisés pour la valorisation du best-estimate des contrats d'épargne en euro doivent être cohérents avec les prix du marché (Market-Consistent). Une évaluation en valeur de marché consiste à valoriser des grandeurs d'intérêt en se référant aux valeurs des actifs et des passifs réellement échangés. L'objectif est de fabriquer une juste valeur qui soit cohérente avec les prix et les risques observables et mesurables sur le marché.

Appliquer une démarche Mark to Market pour évaluer le best-estimate en juste valeur implique de disposer a priori des prix des options et des garanties des polices d'assurance. Cette information n'étant pas observable sur un marché organisé et liquide, le calcul est donc mené dans un cadre Mark-to-Model. Dans ce cadre, le GSE est calibré non pas sur les options et garanties du contrat d'assurance mais sur des produits financiers (caps, floors, swaptions...). Sa qualité est appréciée par sa capacité à reproduire les prix de ces produits financiers. 
Les orientations élaborées par l'EIOPA4 (cf. ACPR [2015] - orientations 55 à 60) présentent un certain nombre de contraintes qu'un GSE doit satisfaire. Il y est notamment précisé que:

- Les instruments financiers retenus pour calibrer les GSE doivent être pertinents eu égard aux caractéristiques des options et garanties financières proposées par l'assureur;

- Les données doivent provenir de marchés financiers qui soient profonds, liquides et transparents. Les résultats fournis par le GSE doivent être cohérents avec les données du marché financier (règlement délégué article 76).

\subsection{La convention de calibrage des modèles de taux sans risque}

L'EIOPA publie tous les mois une courbe des taux sans risque utilisée pour l'actualisation des flux futurs afin d'évaluer le best-estimate dans le référentiel Solvabilité 2 .

Les spécifications techniques QIS [2010] précisent qu'un calibrage market-consistent doit se présenter comme suit (TP.2.97):

- Le modèle d'actifs doit être calibré pour refléter la nature et la durée des passifs, en particulier les passifs intégrant des garanties et des options;

- Le modèle d'actifs doit être calibré en prenant en compte la courbe de taux sans risque utilisée pour actualiser les flux de trésorerie;

- Le modèle d'actifs doit être calibré à une mesure de la volatilité correctement calibrée.

Par ailleurs, l'article 22 du règlement délégué [2014] précise que le calibrage des paramètres et des scénarios doit être cohérent avec la courbe des taux sans risque pertinents utilisée pour calculer la meilleure estimation visée à l'article 77 , paragraphe 2 , de la directive 2009/138/CE.

Face à ces contraintes réglementaires et en l'absence de prix observables des passifs des contrats d'épargne en euro, une convention de calibrage des taux sans risque s'est établie. Cette convention peut se synthétiser en quatre étapes: 
1. Modèle et instruments financiers dérivés: choix du modèle de taux et choix des produits dérivés pour son calibrage: caps, floors, swaptions...

2. Prix d'exercice et volatilités implicites: choix d'un prix d'exercice et extraction des volatilités de marché. Ces volatilités correspondent aux volatilités implicites des produits dérivés choisis à l'étape 1. Elles sont cohérentes avec la courbe de taux sans risque du marché.

3. Valorisation des produits dérivés en utilisant la courbe publiée par l'EIOPA : utilisation du modèle de Black (si les volatilités sont implicites à un modèle log-normal) ou du modèle de Bachelier (si les volatilités sont implicites à un modèle normal) pour l'évaluation d'un prix des instruments dérivés en utilisant la courbe de taux sans risque publiée par l'EIOPA. Ce sont ces prix qui vont jouer le rôle de "prix de marché" pour calibrer le modèle de taux retenu.

4. Calibrage du modèle de taux retenu en minimisant une distance entre: (1) les prix ré-estimés à partir de la courbe de taux EIOPA et les volatilités de marché et (2) les prix théoriques du modèle de taux.

Notons que la courbe de taux sans risque est fournie par le régulateur, le choix d'un modèle de taux sans risque dans un espace gaussien revient donc à choisir une structure de volatilité.

La courbe des taux sans risque communiquée par l'EIOPA est en général différente de la courbe des taux swap du marché. Ainsi, le processus de calibrage présenté ci-dessus intègre les volatilités implicites mais ne permet pas, par construction, de répliquer les prix de marché des instruments financiers dérivés. Ce processus respecte néanmoins les contraintes réglementaires qui évoquent un calibrage sur les volatilités implicites.

Notons également que la courbe de taux sans risque communiquée par l'EIOPA est une courbe reconstruite et est, de fait, inobservable sur le marché financier.

Enfin, le régulateur ne précise pas la nature des volatilités à retenir dans un environnement à taux négatifs. Comme expliqué dans la section 2.1.1.3 de l'annexe technique, les volatilités implicites aux modèles de Black dépendent d'un facteur de décalage. Ce facteur dépend de la courbe de taux sans risque utilisée dans le modèle de Black pour extraire les volatilités implicites. 


\subsection{Quelques limites de la convention de calibrage des modèles de taux sans risque pour l'évaluation du best-estimate}

Le best-estimate est une grandeur non observée. Le GSE est calibré non pas sur les options et garanties du contrat d'assurance mais sur des produits financiers (caps, floors, swaptions...).

La problématique de génération de scénarios économiques pour la valorisation du best-estimate implique 5 variables:

1. Le prix du produit dérivé: le GSE est calibré sur des produits financiers (caps, floors, swaption...). Se pose alors la question de la cohérence de la structure optionnelle du best-estimate et du produit financier choisi pour le calibrage du GSE. Cette question est abordée dans Armel et Planchet [2018] qui montrent que le best-estimate a une structure optionnelle par cliquets;

2. Le prix d'exercice (strike): les prix des produits dérivés dépendent des prix d'exercice. Afin de proposer un calibrage des GSE cohérent avec la structure optionnelle du best-estimate, ces GSE devraient être calibrés sur des prix d'exercices cohérents avec les seuils d'exercice des options et des garanties du contrat d'épargne en euro. Il serait pertinent donc d'étudier la moneyness du passif (cf. Armel et Planchet [2018] pour une définition de la moneyness du passif)

3. La courbe des taux sans risque est communiquée par l'EIOPA. Il s'agit d'une courbe de taux non observable sur le marché financier et ne faisant l'objet d'aucun échange marchand;

4. Le facteur de décalage est un facteur introduit pour permettre aux modèles log-normaux de prendre en compte des taux négatifs. Cela permet de (1) reproduire les valeurs de marché par le modèle de Black et calibrer les modèle de taux et de (2) diffuser des modèles log-normaux comme le modèle LMM. Le facteur de décalage dépend de la courbe de taux sans risque utilisée dans le modèle, en particulier, de la valeur minimale de cette courbe (cf. section 2.1.1.3 de l'annexe technique); 
5. Les volatilités implicites dépendent du prix des instruments financiers, du prix d'exercice, du taux d'intérêt sans risque et du facteur de décalage (cf. section 2.1.1 de l'annexe technique). Lors du calibrage des modèles de taux destinés à l'évaluation du best-estimate, ce sont ces volatilités implicites qui sont utilisées dans le modèle de Black. Il est noté que:

a. Le paramétrage du modèle de Black par la courbe de taux sans risque EIOPA implique l'introduction d'un facteur de décalage. Afin de garder une certaine cohérence du modèle, ce facteur devrait être identique à celui utilisé pour extraire les volatilités implicites des cours de marché;

b. La cohérence entre la courbe de taux sans risque EIOPA et la volatilité implicite aux prix de marché n'est pas systématique. Les volatilités dépendent en effet de la courbe de taux sans risque utilisée pour les évaluer (cf. section 2.1.1 de l'annexe technique);

c. Les volatilités implicites dépendent du prix d'exercice des instruments financiers utilisés dans le processus de calibrage. La cohérence entre les volatilités implicites et les options et garanties du passif n'est pas systématique.

\section{DÉmARCHE ET CHOIX DE MODÉLISATION}

La section 5 présente une application des développements théoriques documentés dans l'annexe technique et consiste à générer des scénarios économiques pour valoriser des passifs d'épargne en euro dans le référentiel solvabilité 2 .

Une attention particulière est accordée aux modèles de taux. La convention de calibrage présentée dans la section 2.2 est celle qui est retenue dans l'application présentée dans la suite.

Les choix suivants sont retenus:

- Les options retenues pour le calibrage des différents modèles (call, caps et swaption) sont $A T M^{5}$;

- Les volatilités de marché des caps et swaptions utilisées dans le processus de calibrage sont des volatilités log-normales ATM non décalées fournies par Bloomberg (le facteur de décalage est égale à 0); 
- La courbe de taux sans risque retenue pour les processus de calibrage et de simulation est la courbe de taux fournie par l'EIOPA le 31/12/2017;

- L'utilisation de la courbe EIOPA implique la nécessité d'introduire un facteur de décalage non nul pour calibrer et projeter les modèles de taux. L'introduction de ce facteur non nul (alors que l'extraction des volatilités est réalisée avec un facteur de décalage nul) induit un certain biais dans le modèle comme explicité dans la section 2.3:

- Pour les modèles Hull et White et G2++: différents calibrages ont été réalisés correspondant à différents niveaux du facteur de décalage. Ces modèles sont normaux et ne nécessitent pas la définition d'un facteur de décalage pour la diffusion;

- Le modèle LMM a été calibré sur les volatilités de Black sur les maturités ne présentant pas de taux négatifs: aucun facteur de décalage n'a été introduit dans le processus de calibrage;

- La diffusion du modèle LMM nécessite la définition d'un facteur de décalage. Plusieurs facteurs de décalage ont été testés;

- Trois niveaux du facteur de décalage sont testés: 0,4\%, 1\% et $2 \%$. La valeur de $0,4 \%$ correspond à la valeur absolue arrondie du taux minimal de la courbe de taux sans risque EIOPA le 31 décembre 2017.

- Pour la projection d'investissements en actions et en immobilier, nous avons retenu des modèles de Black-Scholes à volatilités constantes:

- La volatilité implicite à un investissement en actions est calibrée sur la volatilité implicite du call ATM sur le CAC 40 de maturité 3 ans;

- La volatilité d'un investissement en immobilier correspond à la volatilité historique des rendements de l'indice des prix de vente des logements anciens publié par l'INSEE' 6 .

Une synthèse des choix retenus pour la construction des générateurs de scénarios économiques est présentée dans la Figure 2. 
FIGURE 2 Choix de modélisation

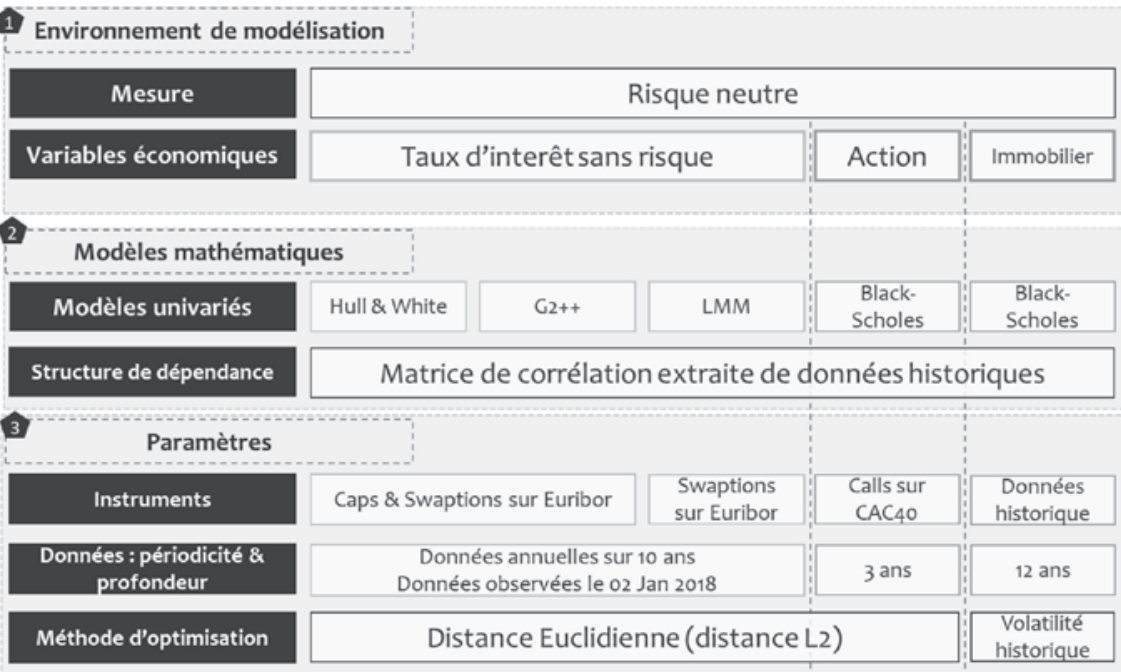

\section{Présentation des données des modèles de tauX}

\subsection{Analyse des taux sans risque du marché et de l'EIOPA}

La courbe de taux sans risque utilisée dans la phase de calibrage et de simulation est la courbe de taux sans risque à terme communiquée par l'EIOPA au 31/12/2017. Nous observons notamment que le taux à terme est négatif sur les trois premières années.

FIGURE 3 Taux sans risque EIOPA sans volatility adjustment au 31 décembre 2017

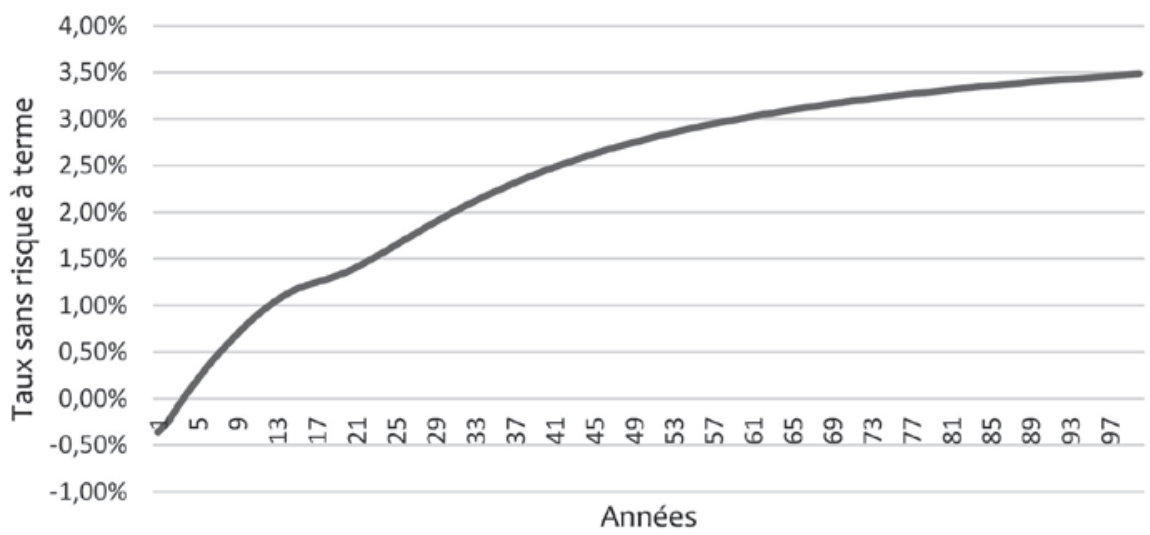


Afin de disposer d'ordre de grandeurs historiques sur l'évolution des taux sans risque historiques et de leur volatilité, nous présentons dans la suite un graphique reprenant l'évolution du TEC 10 et un graphique présentant la volatilité historique par maturité du TEC ${ }^{7}$.

FIGURE 4 Évolution du TEC 10 entre décembre 2004 et décembre 2017

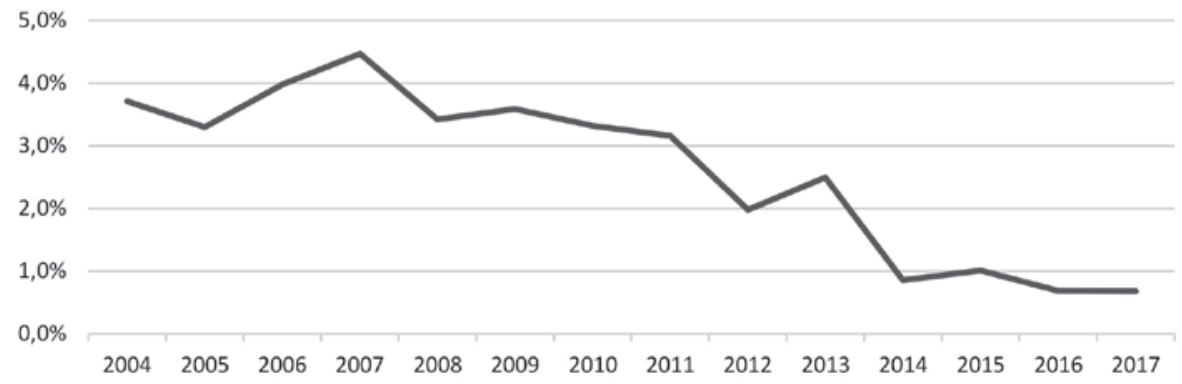

Nous observons une tendance baissière du taux TEC 10 avec un minimum historique en 2017.

FIGURE 5 Volatilités historique par maturité du TEC sur la période 2006-2017

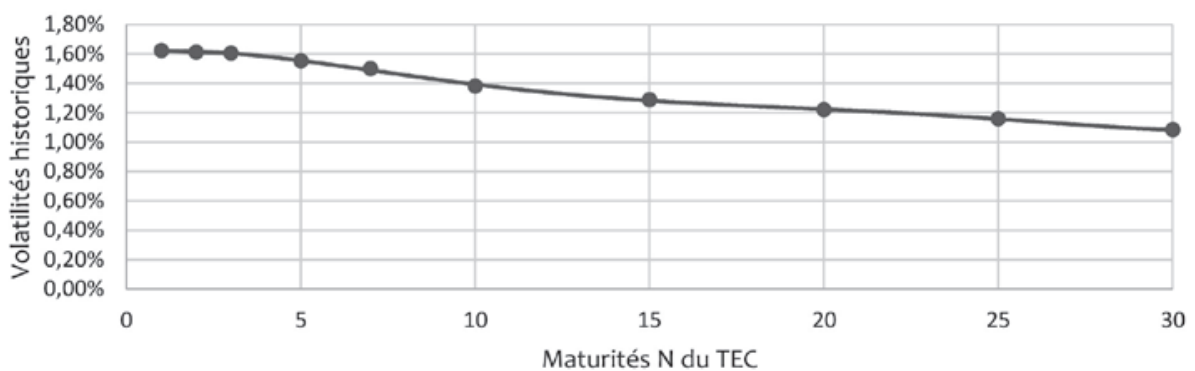

Nous observons que la courbe de volatilité historique par maturité des TEC sur la période allant de décembre 2006 à décembre 2017 est décroissante en fonction de la maturité. La volatilité à une maturité donnée $\mathrm{N}$ est calculée comme l'écart-type des taux historiques à maturité $\mathrm{N}$ sur la période d'observation. 


\subsection{Volatilités implicites des caps et des swaptions}

Les graphiques ci-après sont construits à partir de volatilités log-normales implicites aux prix de marché de caps et de swaptions sur le taux Euribor 3 mois. Ces volatilités sont non-décalées (facteur de décalage $=0$ ). Les données ont été extraites de Bloomberg le 02 janvier 2018.

Le graphique suivant présente la nappe de volatilité log-normale implicite aux prix de caps sur Euribor observés au 02 janvier 2018 (prix d'exercice, maturité et volatilité).

FIGURE 6 Nappe de volatilité log-normale implicite aux prix de caps au 02 janvier 2018

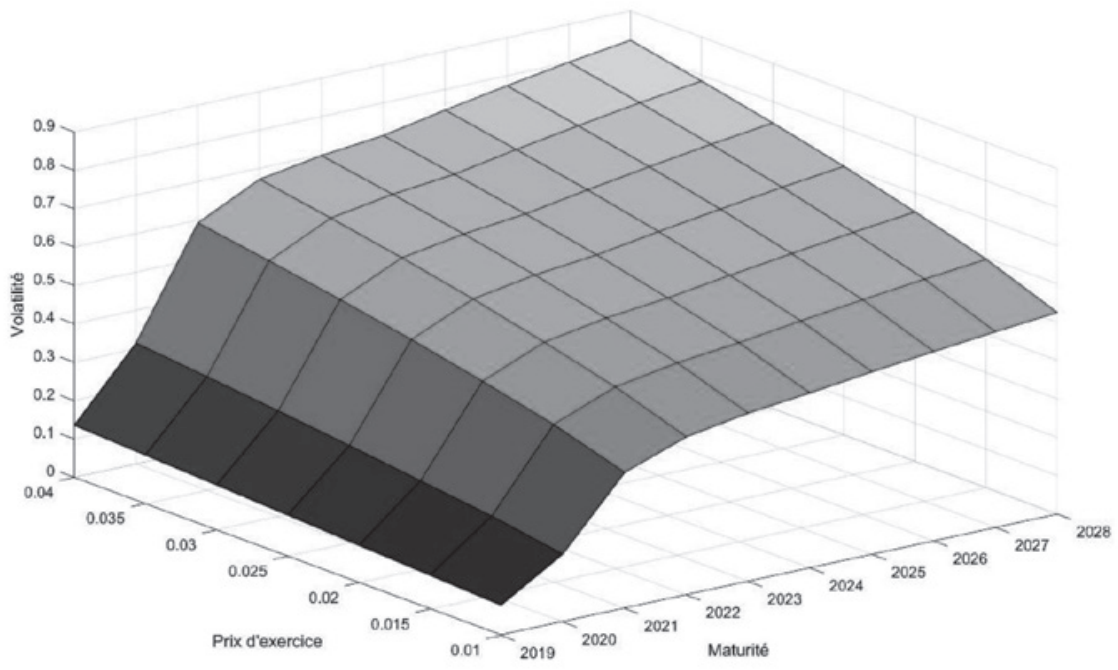

Le graphique suivant présente la volatilité log-normale du cap ATM (At The Money) sur Euribor 3 mois observée au 02 janvier 2018. 
FIGURE 7 Volatilité log-normale du cap ATM observé au 02 janvier 2018

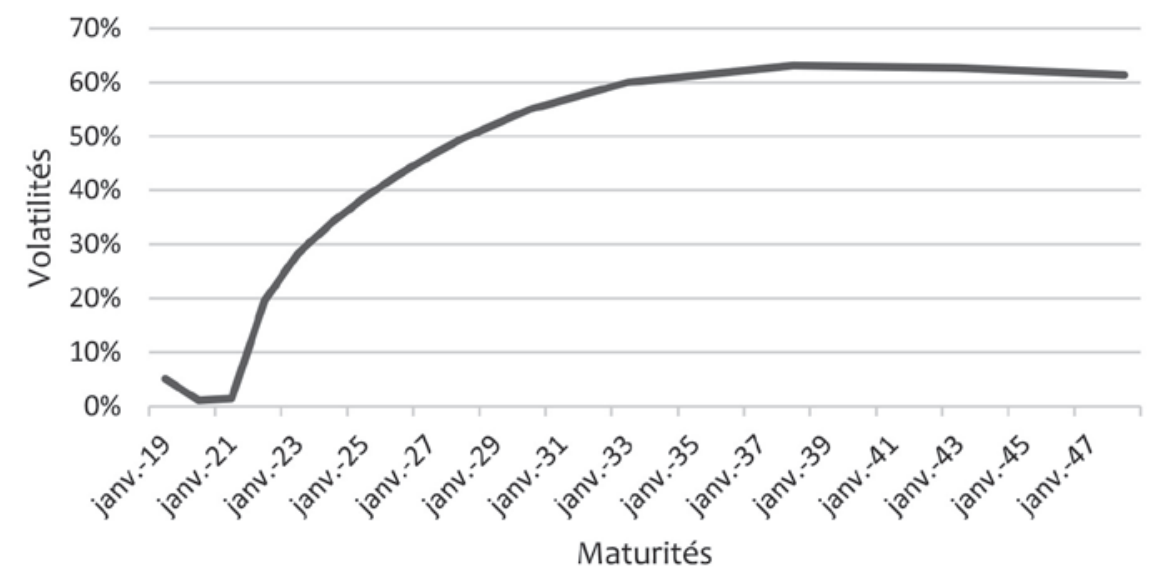

Le graphique suivant présente la nappe de volatilité log-normale implicite aux prix d'une swaption ATM observée au 02 janvier 2018 (dates d'exercice, tenors $^{8}$ et volatilités).

FIGURE 8 Nappe de volatilité log-normale implicite aux prix d'une swaption ATM au 02 janvier 2018

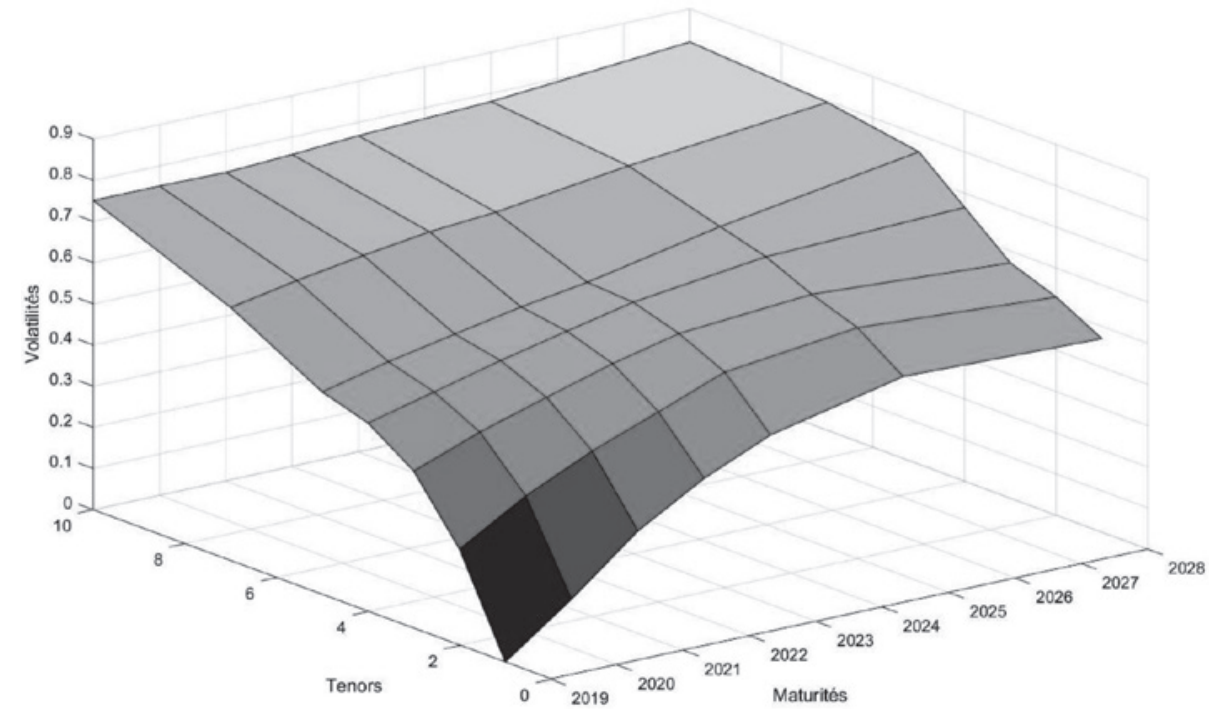

Nous notons que les volatilités implicites log-normales ont globalement une allure croissante en fonction des maturités. 


\section{Calibrage et simulation}

\subsection{Analyse des prix de Black}

Les Figure 9 et Figure 10 montrent que, en gardant les volatilités de marché constantes en fonction du facteur de décalage, les prix de caps pour la maturité 5 ans et de swaptions ATM de maturité 1 an, évalués par le modèle de Black, sont croissants en fonction du facteur de décalage. Rappelons que si les volatilités implicites dépendent du facteur de décalage, les prix de Black sont par construction constants en fonction du facteur de décalage (cf. section 2.1.1.3 de l'annexe technique).

Nous avons réalisé plusieurs tests sur d'autres maturités et le constat est le même: lorsque que l'on fait varier uniquement le facteur de décalage, sans faire varier les volatilités implicites, les prix recalculés par le modèle de Black sont croissants en fonction du facteur de décalage. Nous n'avons pas développé dans ce papier une analyse quantitative pour appuyer ce constat.

FIGURE 9 Évolution des prix de caps évalués par le modèle de Black en fonction du facteur de décalage pour la maturité 5 ans

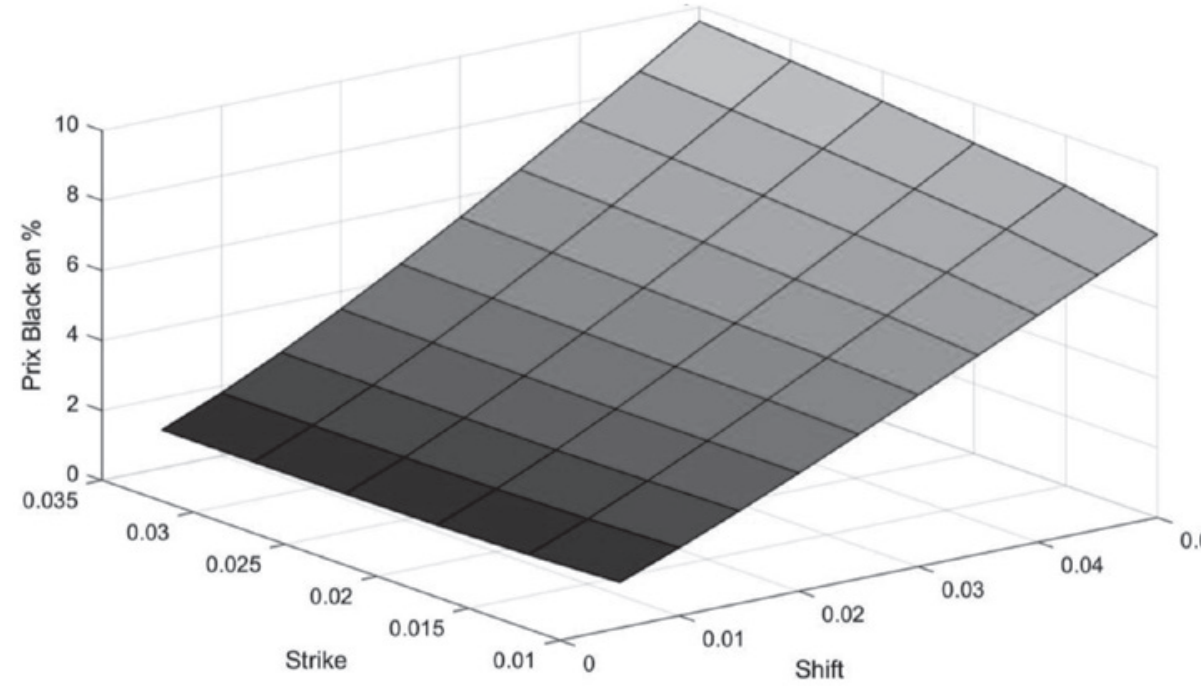




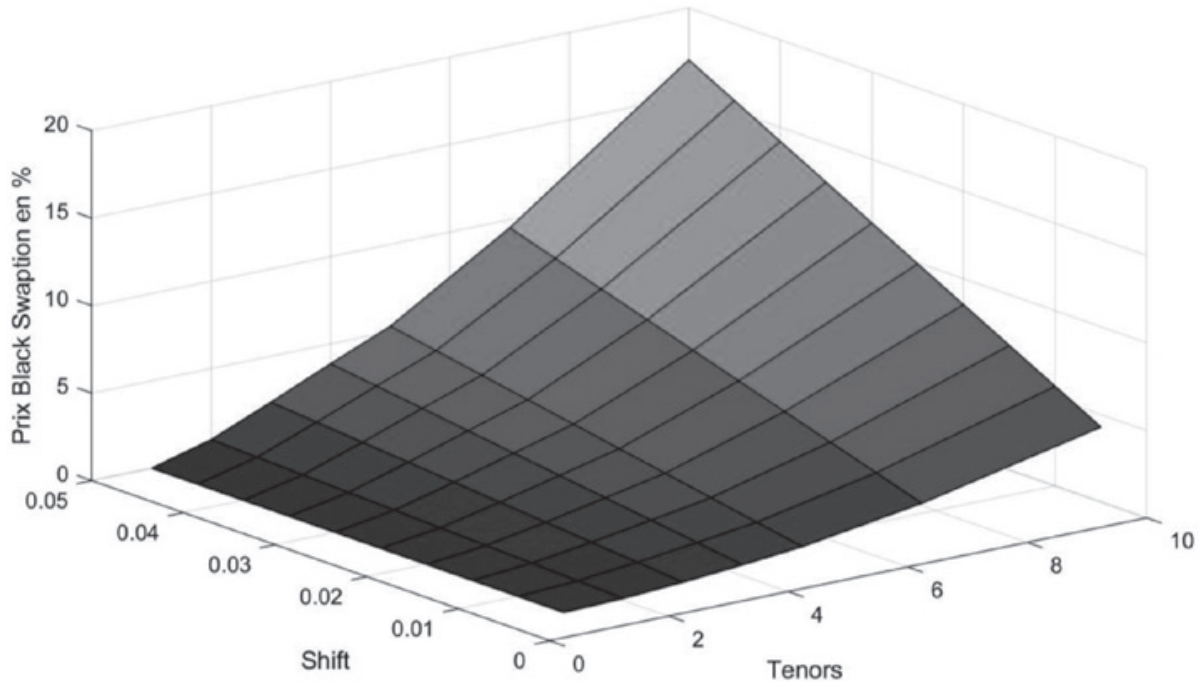

L'introduction d'un facteur de décalage dans le modèle de Black implique une surestimation du prix de marché du produit dérivé. Cela implique que le calibrage des modèles de taux sur des prix recalculés par le modèle de Black décalé (à volatilité non-décalée) introduit une surestimation de la volatilité de ces modèles de taux. Les modèles ont logiquement tendance à "compenser» la surestimation du prix par une augmentation de la volatilité.

\subsection{Modèle Hull \& White}

Le Tableau 1 présente les résultats du calibrage du modèle Hull \& White sur les données de caps et de swaptions présentées ci-dessus.

Nous notons que le paramètre de volatilité locale augmente avec la valeur du facteur de décalage utilisé pour reproduire les prix de marché par le modèle de Black. 


\section{TABLEAU 1 Résultats du calibrage du modèle Hull \& White}

\begin{tabular}{|c|c|c|c|c|}
\hline INDICE & $\begin{array}{l}\text { DÉCALAGE DU } \\
\text { MODÉLE BLACK }\end{array}$ & a & $\sigma$ & $\begin{array}{c}\text { ERREUR TOTALE } \\
\text { AU CARRÉ RELATIVE }\end{array}$ \\
\hline HW 1 (Caps) & $0,40 \%$ & $0,97 \%$ & $0,53 \%$ & $2,71 \%$ \\
\hline HW 2 (Caps) & $1,00 \%$ & $1,00 \%$ & $0,80 \%$ & $2,98 \%$ \\
\hline HW 3 (Caps) & $2,00 \%$ & $1,04 \%$ & $1,25 \%$ & $2,92 \%$ \\
\hline HW 4 (Swaption) & $0,40 \%$ & $0,07 \%$ & $1,27 \%$ & $7,32 \%$ \\
\hline HW 5 (Swaption) & $1,00 \%$ & $0,10 \%$ & $1,62 \%$ & $8,48 \%$ \\
\hline HW 6 (Swaption) & $2,00 \%$ & $0,01 \%$ & $2,20 \%$ & $10,43 \%$ \\
\hline
\end{tabular}

L'erreur totale est calculée comme la somme des erreurs au carré (fonction objectif) divisée par la somme des prix de Black au carré.

La Figure 11 présente la qualité du calibrage du modèle de Hull \& White aux prix de marché évalués par le modèle de Black décalé pour un facteur de décalage égal à $1 \%$. Nous notons que la qualité de reproduction des prix de caps est moins bonne que le modèle G2++ dont les résultats de calibrages sont présentés dans la suite.

FIGURE 11 Qualité de reproduction des prix de caps par le modèle de Hull \& White

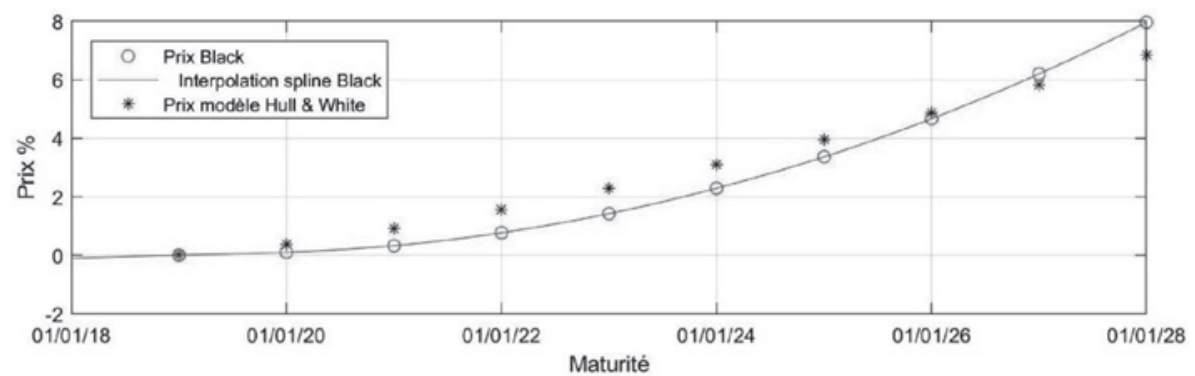

La Figure 12 présente les dynamiques des espérances des taux à terme diffusées par le modèle Hull \& White pour les différents calibrages présentés dans le Tableau 1. Ces espérances sont calculées comme les moyennes, à chaque pas de temps, de 10000 simulations. Nous n'avons pas besoin de simulations pour calculer ces espérances, l'idée est de présenter des indicateurs déduits des trajectoires destinées aux simulations du best-estimate épargne en $€$. 
Nous observons que les espérances peuvent s'écarter significativement de la courbe de taux sans risque centrale. Ce constat est cohérent avec l'expression du taux à terme présenté dans la section 2.1.2 de l'annexe technique: la convergence de l'espérance des taux à terme vers les taux sans risque dépend des paramètres du modèle et notamment de la vitesse de convergence.

FIGURE 12 Convergence des moyennes des différents calibrages du modèle Hull \& White

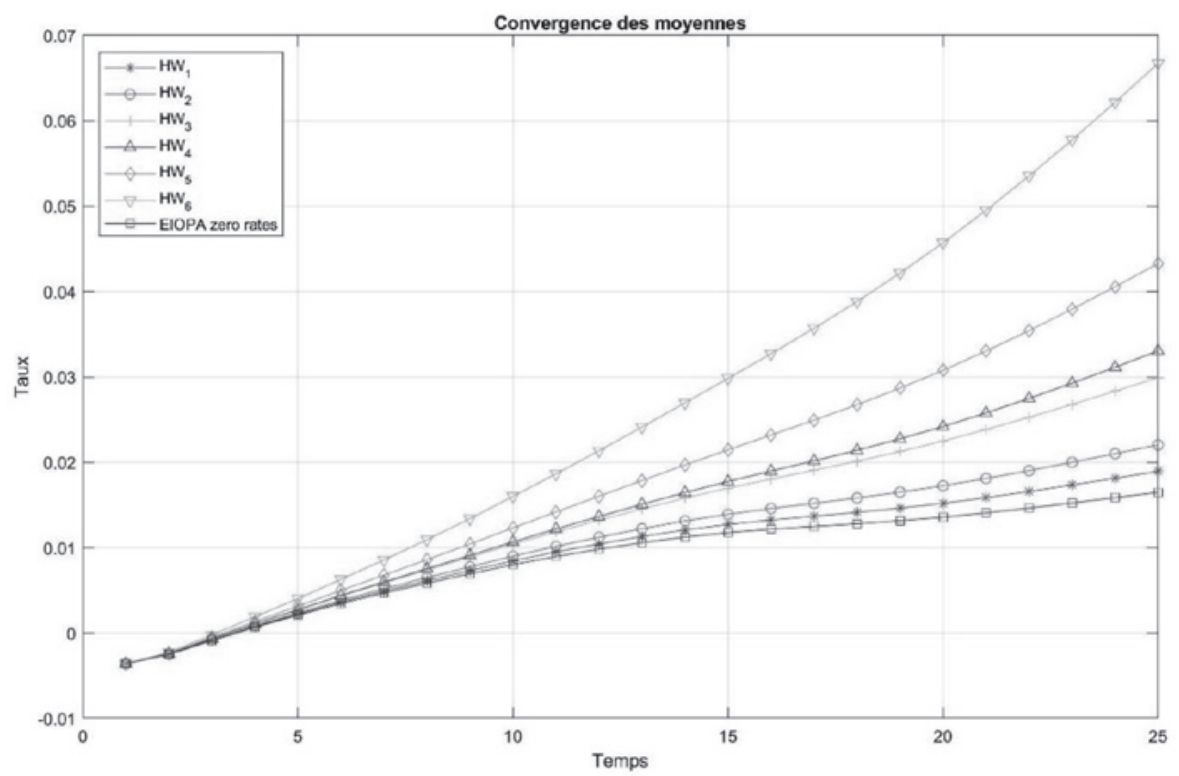

La Figure 13 présente l'évolution des volatilités des taux à terme simulés à chaque pas de temps. Nous constatons que:

- Les écarts entre les espérances et les taux sans risque EIOPA sont d'autant plus importants que les volatilités sont importantes;

- Les volatilités sont croissantes en fonction de la maturité. La monotonie de la volatilité anticipée du modèle en fonction de la maturité est donc différente de celle de la volatilité historique (cf. section 4.1).

Pour chaque type de produit (caps et swaptions), nous notons que la volatilité est croissante en fonction du facteur de décalage. En effet, le prix de Black étant croissant en fonction du facteur de décalage, le modèle de Hull \& White "compense" cette hausse de prix par une hausse de la volatilité. 


\section{FIGURE 13 Courbes de volatilité des différents calibrages}

du modèle Hull \& White

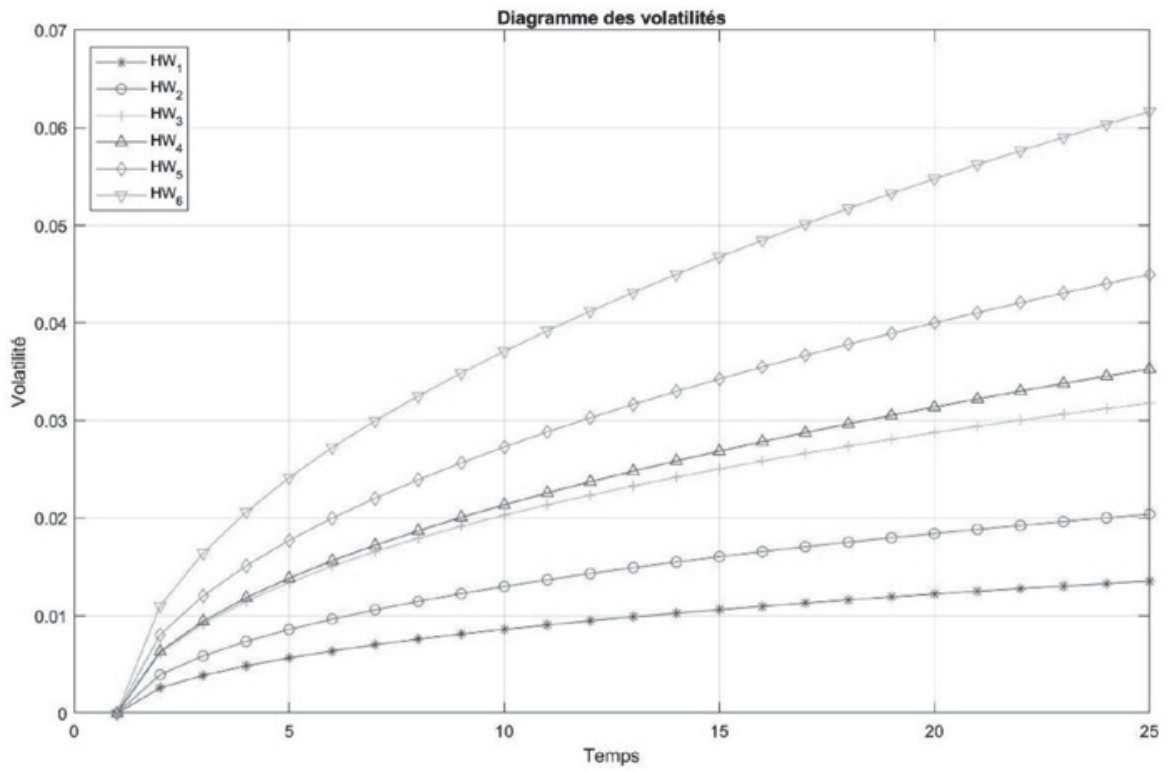

\subsection{Modèle G2++}

Le Tableau 2 présente les résultats du calibrage du modèle G2++ sur les données de caps et de swaptions présentées ci-dessus.

\section{TABLEAU 2 Résultats du calibrage du modèle G2++}

\begin{tabular}{|c|c|c|c|c|c|c|c|}
\hline INDICE & $\begin{array}{l}\text { DÉCALAGE } \\
\text { DE MODĖLE } \\
\text { BLACK }\end{array}$ & a & b & $\sigma$ & $\eta$ & $\rho$ & $\begin{array}{l}\text { ERREUR TOTALE } \\
\text { AU CARRÉ } \\
\text { RELATIVE }\end{array}$ \\
\hline G2 1 (Caps) & $0,40 \%$ & $1,48 \%$ & $0,50 \%$ & $26,02 \%$ & $25,64 \%$ & $-99,99 \%$ & $0,009 \%$ \\
\hline G2 2 (Caps) & $1,00 \%$ & $5,14 \%$ & $0,03 \%$ & $7,50 \%$ & $7,07 \%$ & $-100,00 \%$ & $0,005 \%$ \\
\hline G2 3 (Caps) & $2,00 \%$ & $8,39 \%$ & $4,36 \%$ & $17,46 \%$ & $16,76 \%$ & $-99,99 \%$ & $0,012 \%$ \\
\hline G2 4 (Swaption) & $0,40 \%$ & $11,47 \%$ & $9,04 \%$ & $24,20 \%$ & $23,22 \%$ & $-99,99 \%$ & $0,17 \%$ \\
\hline G2 5 (Swaption) & $1,00 \%$ & $11,49 \%$ & $8,90 \%$ & $26,50 \%$ & $25,67 \%$ & $-99,80 \%$ & $0,21 \%$ \\
\hline G2 6 (Swaption) & $2,00 \%$ & $11,71 \%$ & $8,38 \%$ & $25,59 \%$ & $24,97 \%$ & $-99,97 \%$ & $0,29 \%$ \\
\hline
\end{tabular}


Nous notons que les paramètres de convergence et de volatilité des modèles calibrés sur les caps sont significativement hétérogènes. Ce qui ne permet pas une interprétation de la variation de ces paramètres en fonction du facteur de décalage.

Ces paramètres sont cependant cohérents avec la fonction objectif du calibrage consistant à minimiser la distance euclidienne entre les prix de marché et les prix du modèle G2++. Les erreurs quadratiques sont d'ailleurs assez faibles. La Figure 14 illustre par ailleurs la bonne qualité du calibrage du modèle 2 .

FIGURE 14 Qualité de reproduction des prix de caps par le modèle de $G 2++(G 22$, décalage $=1 \%)$

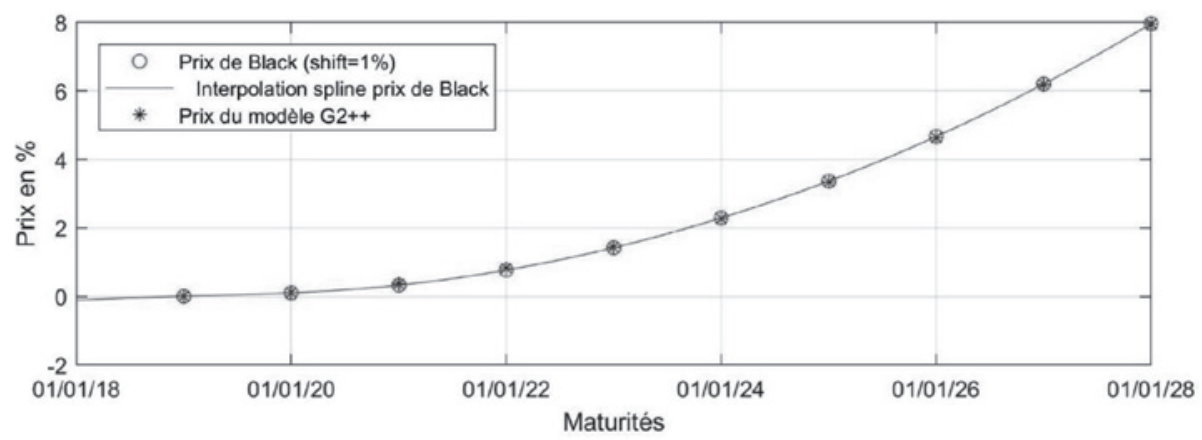

La Figure 15 présente les dynamiques des espérances des taux à terme diffusées par le modèle G2++ pour les différents calibrages présentés dans le Tableau 2 (10 000 simulations sont générées).

Nous observons que les espérances peuvent s'écarter significativement de la courbe de taux sans risque centrale. Comme pour le modèle Hull \& White, la convergence de l'espérance des taux à terme vers les taux sans risque dépend des paramètres du modèle et notamment des vitesses de convergence. La courbe de taux sans risque espérée à la sortie du modèle peut s'écarter donc significativement de la courbe sans risque EIOPA. 
FIGURE 15 Convergence des moyennes des différents calibrages du modèle G2++

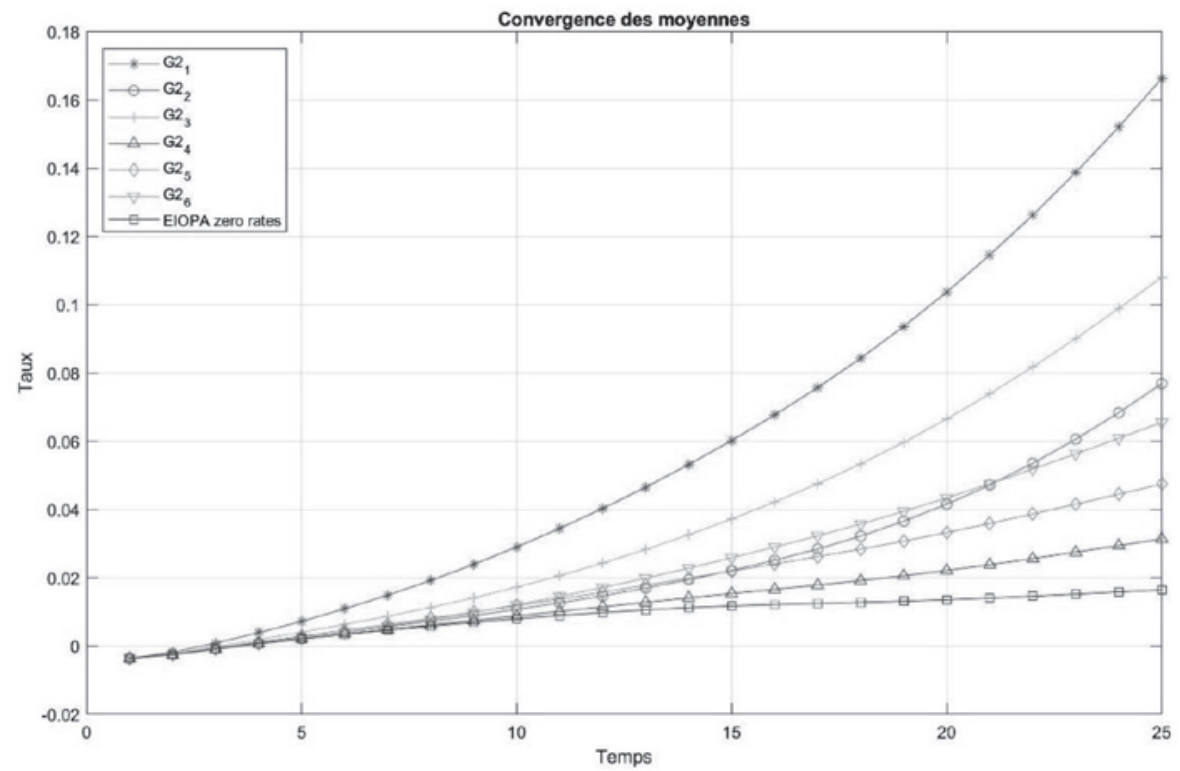

La Figure 16 présente l'évolution des volatilités des taux à terme simulés à chaque pas de temps. Nous constatons que:

- Les écarts entre les espérances et les taux sans risque EIOPA sont d'autant plus importants que les volatilités sont importantes;

- Les volatilités sont croissantes en fonction de la maturité. La monotonie de la volatilité anticipée du modèle en fonction de la maturité est donc différente de celle de la volatilité historique (cf. section 4.1).

Pour les modèles G2++ calibrés sur les swaptions, nous notons que la volatilité est croissante en fonction du facteur de décalage. Pour les courbes de volatilités des modèles G2++ calibrés sur les caps nous notons que la courbe du modèle 2 est inférieure à celle du modèle 3 . Cependant, la courbe de volatilité du modèle 1 domine toutes les autres courbes ce qui limite l'interprétation. Les courbes de volatilité sont en effet difficilement comparables car les paramètres de calibrage sont assez hétérogènes comme expliqué ci-dessus. 
FIGURE 16 Courbes de volatilité des différents calibrages du modèle G2++

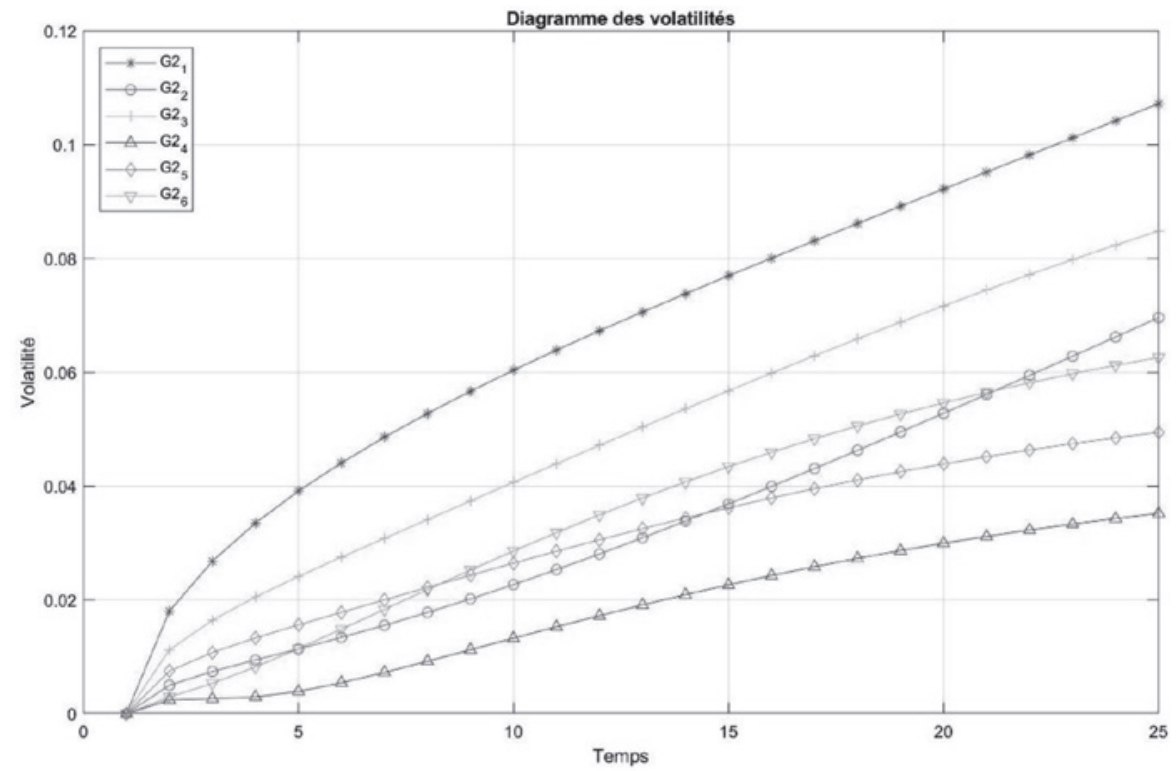

\subsection{Modèle LMM}

5.4.1 Étude du modèle LMM dans un cadre market-consistent

Le Tableau 3 présente les résultats du calibrage du modèle LMM sur les données de swaptions présentées ci-dessus.

\section{TABLEAU 3 Résultats du calibrage du modèle LMM}

\section{FACTEUR \\ DE DÉCALAGE \\ a

b \\ d \\ Beta AU CARRÉ RELATIVE}

\section{$0,00 \%$}

\section{$18,85 \%$}

$0,19 \%$

$7,45 \%$

$0,01 \% \quad 0,10 \%$

$1,19 \%$

Le calibrage est réalisé sans introduire de facteur de décalage en ne retenant que les volatilités de marché correspondant aux taux positifs.

Sur la base de ce calibrage, nous avons diffusé le modèle LMM en introduisant trois niveaux de décalage: le facteur de décalage du modèle LMM 1 est $0,4 \%$, le facteur de décalage du modèle LMM 2 est $1 \%$ et le facteur de décalage du modèle LMM 3 est $2 \%$. 
La Figure 17 présente les dynamiques des espérances des taux à terme diffusés (10 000 simulations sont générées). Sur cette figure, les dynamiques des modèles LMM 2 et LMM 3 sont tronquées et l'horizon de projection est de 10 ans pour le modèle LMM 1 car les espérances de ces modèles commencent à diverger significativement sur les maturités non-représentées.

\section{FIGURE 17 Convergence des moyennes des modèles LMM} décalés market-consistent

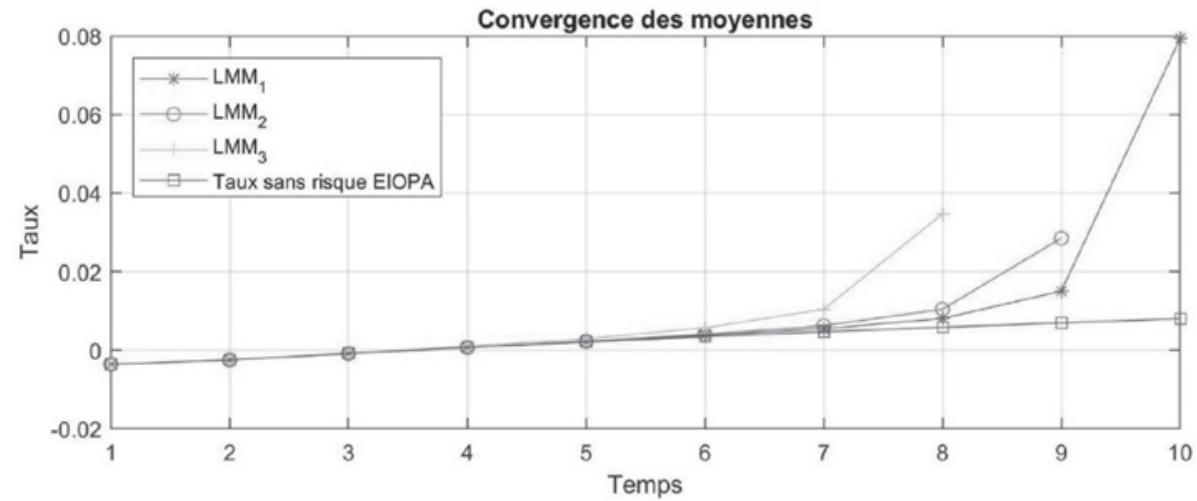

Les courbes de volatilités des modèles LMM décalés sont également divergentes. La Figure 18 présente ces courbes sur les maturités où celles-ci restent comparables.

FIGURE 18 Courbes de volatilité des modèles LMM décalés market-consistent

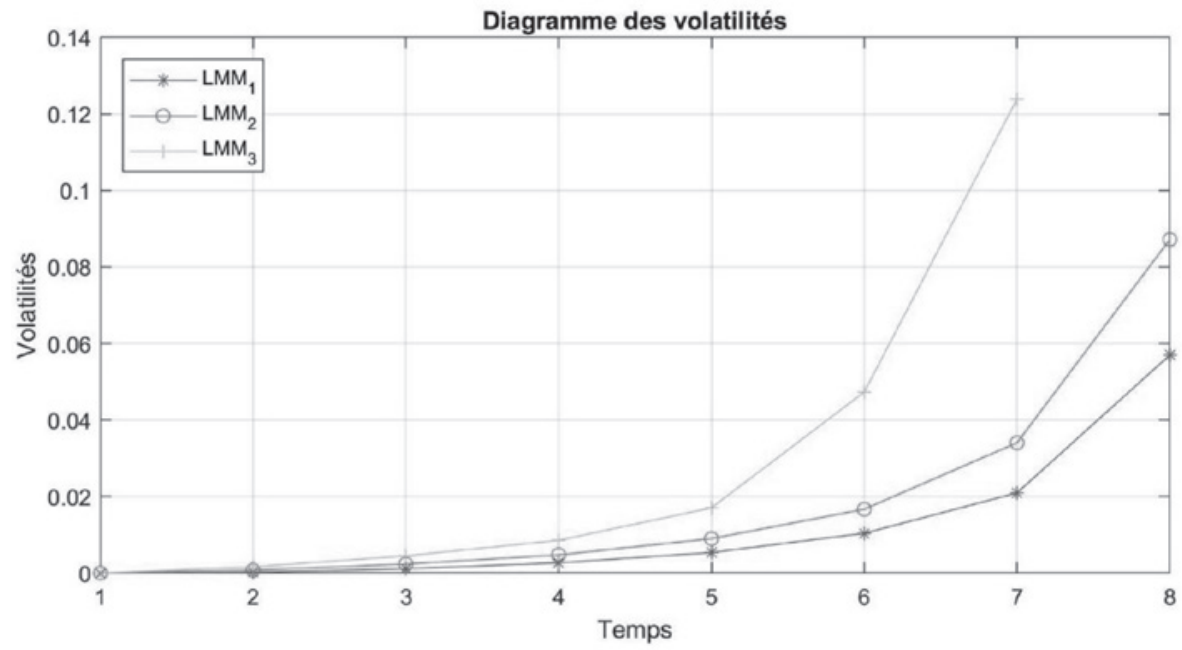


Le modèle LMM calibré sur les swaptions diverge, et ce, même avec le plus petit facteur de décalage admissible pour contourner la contrainte des taux négatifs. Ce calibrage, bien que market consistent, ne peut être retenu dans l'état pour la valorisation des passifs des contrats d'épargne en euro.

Notons que la vitesse de divergence des modèles LMM présentés est croissante en fonction du facteur de décalage.

\subsection{2 Étude du modèle LMM dans un cadre non market-consistent}

Nous nous sommes appuyés sur le calibrage market-consistent réalisé dans la section 5.4.1 pour proposer un paramétrage du modèle LMM convergent. Ce paramétrage n'est de fait pas market-consistent.

Comme présenté dans le Tableau 4, nous avons augmenté la vitesse de convergence de la volatilité de Rebonato et le niveau de la limite asymptotique (cf. section 2.1.4.4 de l'annexe technique pour plus de détails).

\section{TABLEAU 4 Paramétrage convergent du modèle LMM}

\begin{tabular}{|c|c|c|c|c|}
\hline a & b & C & d & Beta \\
\hline $18,85 \%$ & $0,19 \%$ & $20,00 \%$ & $1,00 \%$ & $0,10 \%$ \\
\hline
\end{tabular}

FIGURE 19 Convergence des moyennes des modèles LMM décalés et reparamétrés

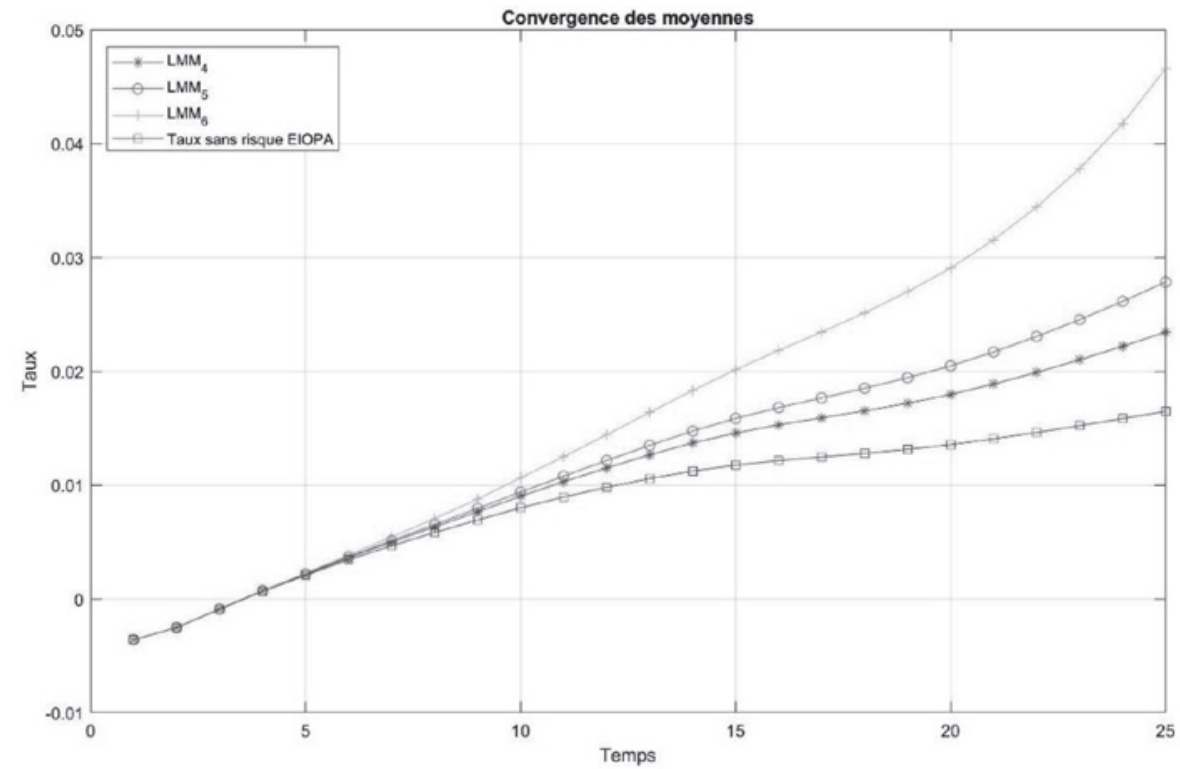


Sur la base de ce paramétrage, nous avons diffusé le modèle LMM en introduisant trois niveaux de décalage: le facteur de décalage du modèle LMM 4 est $0,4 \%$, le facteur de décalage du modèle LMM 5 est $1 \%$ et le facteur de décalage du modèle LMM 6 est $2 \%$.

Nous observons que:

- Les espérances restent dans des niveaux comparables à ceux observés pour les modèles Hull \& White et G2++;

- Certains écarts entre les courbes de taux espérées et la courbe de taux sans risque EIOPA sont significatifs;

- Ces écarts sont plus importants quand le facteur de décalage est plus significatif. Cela se confirme d'ailleurs dans la Figure 20: les volatilités sont croissantes en fonction du facteur de décalage;

- Les volatilités sont croissantes en fonction de la maturité. La monotonie de la volatilité anticipée du modèle en fonction de la maturité est donc différente de celle de la volatilité historique (cf. section 4.1).

\section{FIGURE 20 Courbes de volatilité des modèles LMM} décalés et re-paramétrés

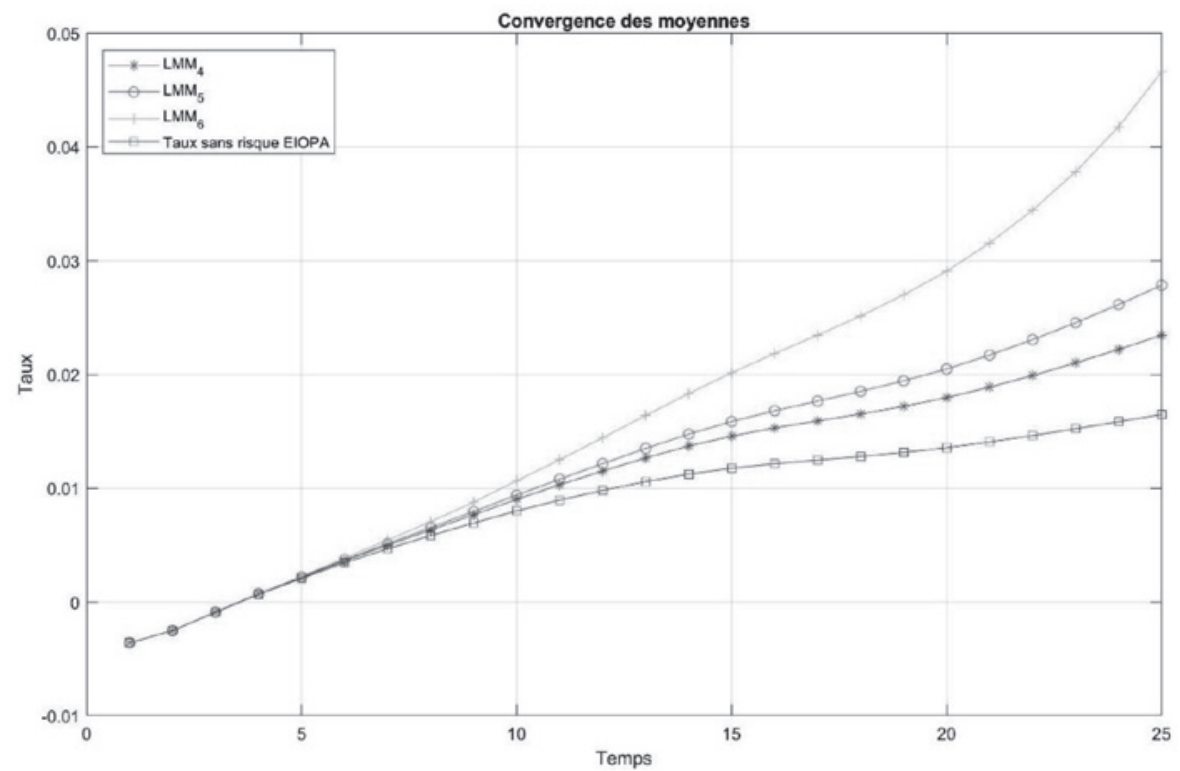




\subsection{Structure de dépendance et modèles actions et immobilier}

Nous avons proposé dans la section 2.3 de l'annexe technique une approche de simulation de l'indice actions et de l'indice immobilier conditionnellement aux trajectoires du taux forward un an avec des matrices de corrélations historiques. Deux matrices ont été estimées: une matrice cohérente avec les modèles Hull \& White et G2++ et une matrice cohérente avec le modèle LMM. Les taux historiques utilisés pour construire ces matrices sont les taux Euribor 12 mois.

\section{TABLEAU 5 Matrices de corrélations}

\begin{tabular}{cc|c|c|}
\hline MATRICE 1 : H \& W ET G2++ & TAUX & \multicolumn{1}{c|}{ ACTION-TAUX } & IMMOBILIER-TAUX \\
\hline TAUX & $100,0 \%$ & $-53,7 \%$ & $-3,9 \%$ \\
ACTION-TAUX & $-53,7 \%$ & $100,0 \%$ & $11,2 \%$ \\
IMMOBILIER-TAUX & $-3,9 \%$ & $11,2 \%$ & $100,0 \%$ \\
\hline MATRICE 2 : LMM & TAUX & ACTION-TAUX & IMMOBILIER-TAUX \\
TAUX & $100,0 \%$ & $-50,4 \%$ & $+3,9 \%$ \\
ACTION-TAUX & $-50,4 \%$ & $100,0 \%$ & $11,2 \%$ \\
IMMOBILIER-TAUX & $+3,9 \%$ & $11,2 \%$ & $100,0 \%$ \\
\hline
\end{tabular}

La volatilité de l'indice immobilier correspond à la volatilité historique des rendements de l'indice des prix de vente des logements anciens publiés par l'INSEE ${ }^{9}$ de décembre 2006 à décembre 2016. Cette volatilité est de $3,5 \%$.

La volatilité du modèle actions est supposée indépendante du temps et a été calibrée sur le prix d'un call $A T M$ de maturité 3 ans sur l'indice CAC 40. Une approche Monte-Carlo a été retenue en intégrant les corrélations présentées ci-dessus pour extraire cette volatilité. Les résultats sont présentés dans le Tableau 6 (le facteur de décalage sous-jacent à chaque modèle de taux est égal à $1 \%$ ). 
TABLEAU 6 Volatilités implicites d'un investissement en actions

\begin{tabular}{c|c} 
MODĖLE DE TAUX & VOLATILITÉ \\
HW 2 & $10,5 \%$ \\
\hline G2++ 2 & $10,6 \%$ \\
\hline LMM 2 & $10,0 \%$ \\
\hline
\end{tabular}

La Figure 21 et la Figure 22 illustrent la diffusion du modèle actions avec le modèle Hull \& White. La courbe de volatilité du logarithme du prix du modèle actions est cohérente avec l'évolution de la volatilité totale en racine carrée du temps.

FIGURE 21 Moyenne et quantiles du modèle actions diffusé avec le modèle HW 2

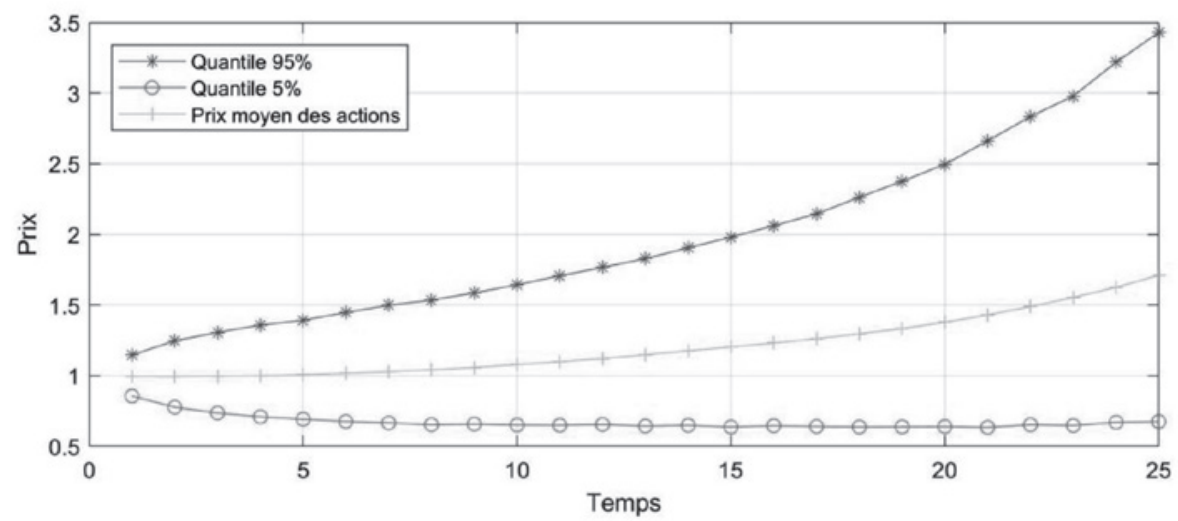

FIGURE 22 Courbe de volatilités du modèle actions diffusé avec le modèle HW 2

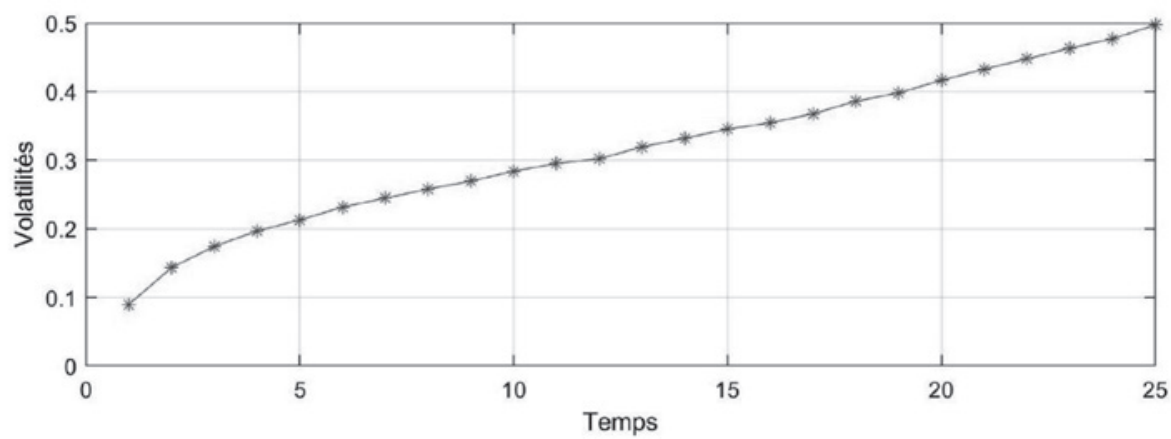




\section{CONCLUSIONS}

La démarche présentée dans ce papier permet de produire des scénarios économiques market-consistent cohérents avec un environnement économique caractérisé par des taux négatifs.

La construction d'un générateur de scénarios économiques est un processus où plusieurs choix sont formulés. Chaque choix a un impact sur les dynamiques simulées des variables d'intérêt.

Aussi, les choix de modélisation et de calibrage doivent, en principe, dépendre de la destination (ou l'usage) du modèle et les hypothèses formulées doivent être confrontées aux réalisations observées.

L'objectivation de ces choix dans le cadre de l'évaluation du bestestimate est limitée car ce dernier n'est pas observable. La démarche de modélisation présentée à la section 2.2 est donc conventionnelle dans la mesure où elle ne peut être validée par une approche statistique basée sur des observations. Cette problématique est discutée dans Armel et Planchet [2018].

Par ailleurs, la market-consistency du GSE destiné à l'évaluation du best-estimate est limitée. Les volatilités de marché dépendent en effet d'une courbe de taux de marché, d'un prix d'exercice et d'un facteur de décalage. Elles ne sont pas systématiquement cohérentes avec la courbe des taux EIOPA et les options et garanties du passif comme discuté dans la section 2.3.

Le choix des modèles mathématiques et des instruments financiers servant à leur calibrage a un impact significatif sur la dynamique des trajectoires simulées et la convergence des moyennes (cf. section 5). Le modèle LMM calibré sur des données de marché au 02/01/2018 présente des divergences significatives des moyennes et des écarttypes. Il ne peut être utilisé en l'état dans le processus d'évaluation du best-estimate. Cette divergence dépend du paramétrage et peut être pilotée, entre autres, par la vitesse de convergence de la volatilité de Rebonato (cf. section 5.4.2).

Une attention particulière doit être accordée à l'introduction des facteurs de décalage dans les processus de calibrage et de simulation des modèles de taux. Quand les volatilités de marché sont non-décalées, ces modèles ont tendance à produire des volatilités croissantes en fonction du facteur de décalage. 
Enfin, la convergence des espérances mathématiques des modèles de taux sans risque dépend des paramètres, notamment des vitesses de convergence (celle de l'espérance du taux court pour les modèles Hull \& White et G2++ et celle de la volatilité pour le modèle LMM). La courbe des taux sans risque espérée et la courbe sans risque EIOPA peuvent présenter des écarts significatifs.

\section{RÉFÉRENCES}

[1] ACPR Banque de France. [2015] «NOTICE Solvabilité II, Provisions techniques (y compris mesures "branches longues»)", Publication et textes de référence.

[2] Règlement délégué [2014] «Commission Delegated Regulation (EU) 2015/35", Official Journal of the European Union.

[3] Armel K., Planchet F., Kamega A. [2011] "Quelle structure de dépendance pour un générateur de scénarios économiques en assurance?», Bulletin Français d'Actuariat, vol. 11, n²2.

[4] Armel K., Planchet F. [2018] «Comment définir la qualité d'un générateur de scénarios économiques destiné à évaluer le best-estimate d'un contrat d'épargne?", Bankers, Markets and Investors, à paraître.

[5] Ballotta L. [2004] "Alternative framework for the fair valuation of participating life insurance contracts", Cass Business School, Actuarial Research Paper $\mathrm{n}^{\circ} 157$.

[6] Baldvinsdóttir E. K., Palmborg L. [2011] «On Constructing a Market Consistent Economic Scenario Generator", Handelsbanken Liv.

[7] Brigo D., Mercurio F. [2007] «Interest Rate Models - Theory and Practice». 2nd Edition. Springer.

[8] Caja A., Planchet F. [2010] «La mesure du prix de marché du risque: quels outils pour une utilisation dans les modèles en assurance?", Assurances et gestion des risques, Vol. 78 (3-4).

[9] Cheng P.K., Planchet F. [2018] «Stochastic Deflator for an Economic Scenario Generator with Five Factors ", Document de travail, ISFA

[10] Da Fonseca J., Grasselli M., Tebaldi C. [2007] «Option pricing when correlations are stochastic: an analytical framework ", Review of Derivatives Research, Volume 10, No 2, Mai (2007).

[11] Dastarac H., Sauveplane P. [2010] «Les déflateurs stochastiques: quelle utilisation en assurance?», Mémoire d'actuaire, ENSAE. 
[12] Djehiche B., Hörfelt P. [2005] «Standard approaches to asset \& liability risk", Supported by the Swedish Insurance Federation. Scandinavian Actuarial Journal. 2005(5): 377-400.

[13] Driessen J., Maenhout P., Vilkov G. [2005] «Option-Implied Correlations and the Price of Correlation Risk ", Advanced Risk \& Portfolio Management Paper.

[14] Errais E., Mauri G., Mercurio F. [2004] «Capturing the Skew in Interest Rate Derivatives: A Shifted Lognormal LIBOR Model with Uncertain Parameters", Working paper.

[15] Haastrecht A. [2010] «Pricing Long-term Options with Stochastic Volatility and Stochastic Interest Rates", Phd Thesis, Published by Wohrmann Print Service, Zutphen, The Netherlands.

[16] Hill J. [2009] "A Note on Implementing LIBOR Market Model", The Numerical Algorithms Group Ltd, Wilkinson House, working paper.

[17] Hull J., White A. [1990] "Pricing interest rate derivative securities", Review of Financial Studies 3, 573-92.

[18] Hull J., [2007] "Options, futures et autres actifs dérivés", PEARSON EDUCATION, Edition 6.

[19] Hull J., White A. [1994] «Numerical Procedures for Implementing Term Structure Models I: Single-Factor Models». The Journal of Derivatives 2, 7-16.

[20] Koivu M., Pennanen T., Ranne A. [2005] «Modeling assets and liabilities of a Finnish pension insurance company: a VEqC approach", Scandinavian Actuarial Journal, 2005(1): 46-76.

[21] Laurent J.P., Norberg R., Planchet F. (editors) [2016] «Modelling in life insurance - a management perspective ", EAA Series, Springer.

[22] Planchet F., Kamega A., Thérond P.E. [2009] «Scénarios économiques en assurance - Modélisation et simulation", Paris : Economica.

[23] QIS5 [2010] «Technical Specifications, Annex to Call for Advice from CEIOPS on QIS5", European Commission.

[24] Rebonato R. [1999] «On the Pricing Implications of the Joint Lognormal Assumption for the Swaption and Cap Market", The Journal of Computational Finance 2, 57-76.

[25] Rebonato R. [2004] «Volatility and correlation the perfect hedger and the fox", 2nd ed. Chichester: John Wiley and Sons Ltd.

[26] Tankov P. [2011] "Calibration de Modèles et Couverture de Produits Dérivés", Université Paris-Diderot (Paris VII). 
[27] Tankov P. [2015] «Surface de volatilité», Université Paris-Diderot (Paris VII).

[28] Vasicek O. [1977] «An Equilibrium Characterization of the Term Structure", Journal of Financial Economics 5, 177-188.

[29] Walker M.B. [2005] «Risk-Neutral Correlations in the Pricing and Hedging of Basket Credit Derivatives ", Journal of Credit Risk.

\section{NOTES}

1. Kamal Armel est actuaire qualifié/certifié et fondateur d'ARMEL Consulting.

Contact: kamal.armel@armelconsulting.fr

2. Frédéric Planchet est Professeur à l'ISFA et actuaire associé à PRIM'ACT. Contact: frederic@planchet.net

3. Le lecteur peut se référer à Armel et Planchet [2018] pour une présentation détaillée du cadre et du processus quantitatif d'évaluation du best-estimate des contrats d'épargne en $€$.

4. European Insurance and Occupational Pensions Authority.

5. Un cap, un floor ou une swaption sont ATM (At The Money) si et seulement si le prix d'exercice s'écrit $K_{\text {ATM }}=\frac{P\left(t, T_{\alpha}\right)-P\left(t, T_{\beta}\right)}{\sum_{j=\alpha+1}^{\beta} \tau_{j} P\left(t, T_{j}\right)}$.

6. https://www.insee.fr/fr/statistiques/series/102770558

7. Rappelons que l'indice quotidien TEC N, Taux de l'Échéance Constante N ans, pour N variant de 1 à 30 , est le taux de rendement actuariel d'une valeur du Trésor dont la durée de vie serait à chaque instant égale à $\mathrm{N}$ années. Ce taux est obtenu par interpolation linéaire entre les taux de rendement actuariels annuels des 2 valeurs du Trésor qui encadrent au plus proche la maturité $\mathrm{N}$.

8. Temps restant jusqu'à la maturité.

9. https://www.insee.fr/fr/statistiques/series/102770558 